On typologies and practical orders in architecture

PALABRAS CLAVE - TIPOLOCÍAS • CANON - ORIENTACIÓN LENGUAJE P PRACTICIDAD

KEYWORDS - TYPOLOGIES · CANON · REFERENCE · LANGUAGE. PRACTICALITY
RESUMEN

La noción de tipología es una de las herramientas fundamentales dentro de las prácticas de conocimiento de la arquitectura. Alude a sus modos de reconocer y ordenar estructuralmente el conjunto de sus obras, pero también es un instrumento útil para distribuir y mantener abiertas sus referencias, de manera de poder integrar nuevos contenidos y cooperar con comunidades epistemológicamente diferentes. Utilizando un enfoque que integra la teorí del proyecto arquitectónico con los estudios sociales de Ciencia y Tecnología, el presente texto describe la noción de tipología a partir de definiciones presentes en la literatura arquitectónica. Se le presenta como una organización canónica de la obra realizada, como una orientación especulativa de diseño, y como un lenguaje flexible interpretativamente que permite entenderse y cooperar con otros. Finalmente se discute esta flexibilidad interpretativa como un signo de la practicidad tanto del concepto como de los modos de orden dentro de la propia arquitectura.

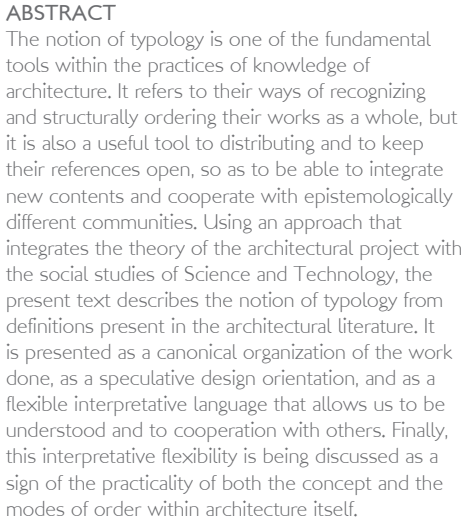

\title{
Sobre las tipologías y los órdenes prácticos en la arquitectura
}

\author{
JORGE VERGARA VIDAL · Universidad de Valparaíso · jorge.vergaravi@uv.cl \\ DANIELA ÁLVAREZ CAMPOS ·Universidad de Santiago·daniela.alvarez@usach.cl \\ DENISSE DINTRANS BAUER · Universidad de Valparaíso·denissedintrans@gmail.com \\ DIEGO ASENJO MUÑOZ · Corporación Municipal de Renca · dmasenjo@uc.cl \\ Fecha recepción: 24 de septiembre 2020 · Fecha aceptación: 14 de noviembre 2020
}

\section{INTRODUCCION: UNA COMUNIDAD Y SUS MODOS DE ORDEN}

Una comunidad es una situación elusiva, planteaba Zigmunt Bauman (2003), porque está basada en un elemento constantemente cambiante: el entendimiento entre quienes la componen. Ferdinand Tönnies (1947) notó tempranamente que este aspecto variaba unido a otro, la voluntad, y que juntos tenían un efecto orgánico en las formas sociales que identificaba como comunidades. Para Tönnies, tanto el entendimiento como la voluntad eran acuerdos sobre los modos de pensar que se implicaban mutuamente, al punto que podían llegar a considerarse como la misma cosa, y pudiendo ser tan fuertes como volubles, tan sólidos como líquidos al decir de Bauman, según estén conducidos por un sentido individual o colectivo de los vínculos.

Según Tönnies (1947), la comunidad original correspondía a una forma social en que la voluntad y el entendimiento individual estaban subjetivamente unidos a la substancia del mundo y se organizaban en torno a elementos esenciales, como la propiedad de la tierra, la ubicación cercana o la sangre compartida entre personas de un mismo grupo. No era vínculo opcional, como podría ocurrir con la elección de un oficio o de una profesión, sino una situación estructural, constitutiva de los actores, que tenía la capacidad de explicar o justificar el sentido de su acción. Sin embargo, dicha situación tenía variaciones en el tiempo, en las que vínculos no esenciales y/o asociaciones electivas adquirían la capacidad de explicar la acción social al generar nuevas comunalidades de prácticas y de actores.

La arquitectura, por ejemplo, no se constituye como una comunidad esencial, pero se organiza como una por medio de una voluntad común, el habitar, y de un entendimiento (técnico) que la hace posible a través del diseño y la construcción. Las prácticas del diseño y la construcción comprenden la voluntad de habitar y expresan el entendimiento espacial, material y social que ello requiere configurando una disciplina, un 'hacer' semejante y común en el tiempo. Las comunidades de prácticas piensan colectivamente: organizan métricas e instrumentos como estrategias para producir similaridades precisas, normativizan los errores y las formas de corregirlos, simetrizan sus modos de hacer inferencias sobre los casos singulares y de ordenar los casos canónicos (Hughes, 2014). A estas tareas la arquitectura dedica sus bienales, congresos, talleres, seminarios, revistas, escuelas; y para esto elabora objetos que le ayudan con su hacer común y similar. Este es el caso de planos, modelos, arquetipos, referentes, estándares y tipologías que traducen esta disciplina de las prácticas en un modo de pensar materialmente realizado (Hughes, 1996; Ingold, 2017). 
Como sostiene Laurent Thevenot (2019), estas formas comunes son parte de un aspecto central de la política del colectivo: permiten a los actores entenderse entre sí y componer el bien de la comunidad. Presentan un código y un formato común aceptado entre ellos, que les hace sentido y les permite comprometerse en la cooperación a pesar de sus diferencias. Operan a la manera de reglas y programas de similitudes y diferencias que mantienen una comunidad compuesta y conflictiva, resolviendo qué tipos de desacuerdos se integran y qué tipo de diferencias son legítimas para ésta.

Este enfoque antropotécnico sobre los objetos materiales lleva a reconocer, como indica Ingold (2013), que no solo actuamos sino también pensamos a través de ellos, abre la posibilidad de explorar su rol en las acciones colectivas y, en especial, releva las prácticas materiales de cooperación que permiten a las comunidades actuar y pensar juntas. Ingold discute la idea de una funcionalidad fija e inherente, o de una agencia en los objetos materiales, y sostiene, en cambio, que estos son más bien poseídos por la acción, lo cual determina una persistente relación especulativa con ellos que en estas páginas se denomina también como practicidad.

El presente texto explora la función de esos objetos poseídos por la acción, particularmente de las tipologías, dentro de las prácticas de conocimiento de la arquitectura en tanto un concepto en uso que describe una relación entre forma y programa (CORVI, 1972; Moneo, 1978, 2015; Star y Giesemer, 1989), y no un estudio de rasgos y formas comunes (Guggenheim, 2016; Zayas, 2015). En este sentido, la semiótica material expresada en las tipologías permite a los actores de la comunidad de prácticas de la arquitectura, entenderse y organizarse en torno a los proyectos y las obras; y a su vez, ser entendidos por actores y comunidades de prácticas diferentes sin tener que negociar aspectos esenciales del oficio o de sus obras. De esta manera, las tipologías son útiles para hacer política dentro de la arquitectura, para homologar el conocimiento de sus practicantes y para tomar decisiones entre ellos, y configuran un espacio político, donde es posible entenderse con otras comunidades de prácticas como constructores, desarrolladores inmobiliarios, financistas, usuarios, entre otros, y planificar acciones conjuntas. En especial, interesa discutir su papel como un tipo de técnicas objetuales que facilitan la cohesión de comunidades de prácticas, y dan sustento a la crítica y la innovación dentro de ellas.

Para ello el texto describe tres modos de actuar de la noción de tipologías dentro de la arquitectura: como organización de la obra realizada, como orientación de diseño, y como lenguaje para la cooperación entre comunidades de prácticas diferentes. Estos tres modos no dan cuenta de todas las posibilidades performativas del concepto, pero ayudan a establecer que las tipologías trabajan de más de una manera y en diferentes espacios de prácticas. Su flexibilidad les permite presentarse como un instrumento especulativo suficientemente bueno, tanto para sistematizar el conjunto de las obras de arquitectura, como para hacer conjeturas sobre éstas sin contar, necesariamente, con toda la información para ello. Este aspecto es abordado en la parte final en la que se discute el papel que cumplen las tipologías en la cohesión de la comunidad de prácticas observada, y lo que dicen acerca de la composición del bien (voluntad/entendimiento) que la define y la organiza.

\section{ORDEN CANÓNICO: UNA ORGANIZACIÓN DE LA OBRA REALIZADA}

A lo largo del tiempo, la arquitectura ha desarrollado y utilizado diversos instrumentos para coordinar y estandarizar sus prácticas de manera de constituirse en una comunidad de conocimientos específica. Un buen número de estos objetos no han sido elaborados dentro de esta comunidad, pero han sido asimilados por ésta como parte de sus técnicas. Los planos de obra y de planta urbana tienen orígenes técnicos diversos, especialmente militares (Delfante, 2006); mientras las nociones de escala, volumetría y cálculo estructural fueron desarrolladas primero dentro de las bellas artes y dentro de la ingeniería (de Graaf, 2017). Sin embargo, su uso progresivo en el diseño y la producción de hechos de arquitectura (Durand, 2000; Rossi, 2015), no solo ayudó a replicar aprendizajes y modelos constructivos desde el punto de vista de esta comunidad, sino también facilitó la necesaria cooperación epistemológica con otras comunidades de prácticas involucradas en la construcción de las obras y en el planeamiento de las ciudades.

En la arquitectura, las nociones tipológicas emergen como parte de este instrumental que permite a una comunidad de prácticas producir objetos similares, independiente del tiempo o de la ubicación y, por tanto, acuñar y perfeccionar repertorios socio técnicos adecuados para ello. Constituyen prácticas reflexivas, mediante las cuales dicha comunidad ordena y valora lo realizado singularmente por sus miembros, y lo proyecta como una saber práctico y disciplinar. Por tanto, son útiles para ordenar, clasificar y agrupar la obra construida. Como sostiene Rafael Moneo (1978), preguntarse qué clase de objeto es una obra de arquitectura pasa por preguntarse también qué es un tipo o una tipología dentro de un conjunto de obras marcadas por la singularidad.

Tanto Moneo (1978), como Gulio Carlo Argan (1983), entre otros, establecen en la obra de Quatremere de Quincy, escrita a fines del siglo XVIII, una primera reflexión tipológica que, motivada por la innovación técnica y los cambios sociales, hacía el esfuerzo de sistematizar las formas construidas con el fin de orientar la producción arquitectónica con la lógica de la forma, con base en la razón y el uso de la obra, inspirados en la soluciones materiales de la Naturaleza, algo a lo que también recurre Luis Henry Sullivan (1896) al explicar el sentido funcional de los edificios en altura. La forma sigue a la función, sostiene, tal como ocurre con las entidades orgánicas. Pero, sin duda, son las lecciones de Jean-NicolasLouis Durand (2000) donde se establece, con claridad gráfica, un modo de ordenar las 
prácticas de arquitectura según comunalidades de formas abstractas y distintas a lo observado en lo natural. Durand centra en las necesidades relacionales, el argumento para la composición y disposición de los espacios construidos. Sus dibujos sobre la forma de los edificios, basados en formas cuadrada y circulares compuestas modularmente, representan el primer ejercicio de sistematización de las formas construidas en el pasado, para su utilización sistemática en las prácticas de arquitectura del futuro.

Siguiendo la idea de Durand de que los edificios son la base compositiva de las ciudades, Aldo Rossi (2015) propone que éstos dan origen a un conjunto de clasificaciones diferenciadas de la planta, que constituye un problema diferente a la morfología de las ciudades, pero en relación con ésta, de la misma manera como partes que se relacionan binariamente con un todo. En este sentido, el conjunto de edificios y sus modos de clasificación constituyen "hechos de ciudad", posibles de estudio dentro de una ciencia de las ciudades.

Moneo define tipología como "aquel concepto que describe un grupo de objetos caracterizados por tener la misma estructura formal" (1978: 190). Se trata de un modo de agrupación o clasificación basado en las similitudes estructurales que comparten los objetos (Argan, 1983), una abstracción que permite a la comunidad de prácticas pensar en grupos, en un orden de conglomerados o poblaciones de formas. La base de la noción de tipología responde a la comunalidad de la forma estructural y no, aunque puede coincidir, a la composición material o al destino de la obra.

La idea de tipo en arquitectura apela a un recurso epistemológico utilizado por esta comunidad para discernir las estructuras que ordenan la realidad material en el tiempo (Collado, 2017; Quintanilla, 2018). Dentro de este marco, las nociones de tipología y de estándar operan indistintamente como modos racionalizados de diferenciación y categorización, y como modos de enlace y cohesión entre las prácticas de producción y las
1. Elaboración propia a partir de "Conjuntos de edificios resultantes de las divisiones del cuadrado, el paralelogramo, y sus combinaciones con el círculo" de J.N.L. Durand, 1802.

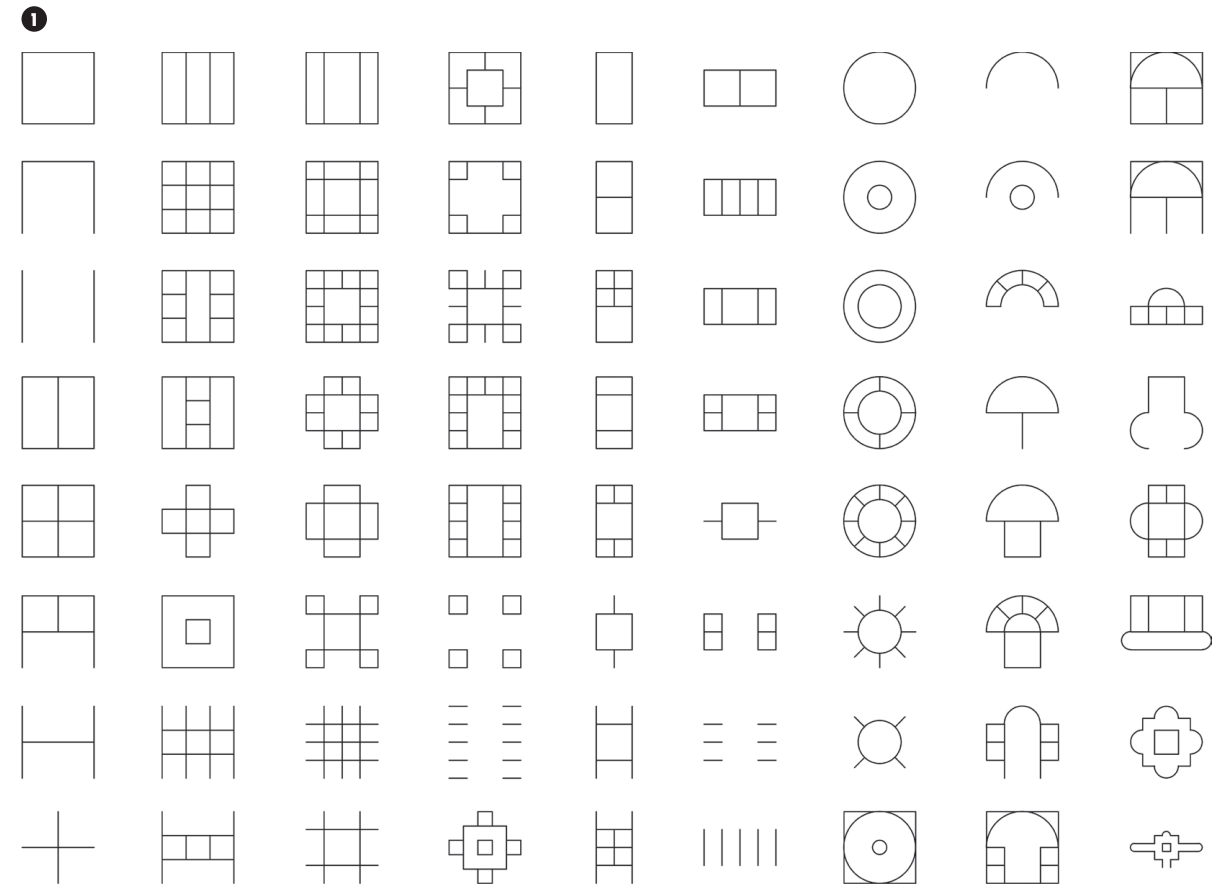

reflexiones sociomateriales de las sociedades sobre la forma construida. En específico, la noción de tipología apela a un modo de clasificación y agrupamiento de un conjunto de obras que presentan una estructura formal similar, como ocurre cuando se diferencia entre los tipos ideales de casas, edificios de altura o blocks.

\section{ORDEN PRÁCTICO: UNA ORIENTACIÓN DE DISEÑO}

Reiner de Graaf (2017), representa el impacto de la obra de Durand en la idea de la caja, como unidad modular básica de la arquitectura moderna. La caja de Durand sostiene, se convierte en una forma tipológica inevitable para las prácticas de arquitectura, una forma compositiva en que geometría, economía y estética se enlazan en un modo de racionalidad replicable, dispuesto para la producción moderna (fordista) de la arquitectura. Constituye una forma abstracta, sin ligamentos a ningún lugar ni a ningún tiempo, rastreable en la obra de Durand y en Viollet-le-Duc, pero aplicable a cualquier escala, en las manzanas de Haussmann o Cerdá, en las casas de Gropius y de Mies van Der Rohe o en la planta libre de Le Corbusier. La caja, sostiene de Graaf, es esencial para la sintaxis disciplinar, "la caja actúa como un contrato; permite que los objetos y los espacios coexistan sin interferir en los asuntos del otro. Cada nueva adición contrario, proporciona una legitimidad cada vez mayor. Se convierte en sinónimo del futuro de la arquitectura" (Ibid.: 82).

Coincidentemente, Giulio Carlo Argán (1983) plantea las tipologías como repertorio arquitectónico que da como resultado ejercicios análogos sobre las formas o sus significados que van más allá de la repetición de un apenas limita el espacio de la siguiente; por el 
2. Tipologías de Vivienda CORVI diseñada entre 1966 y 1971. Fuente: CORVI (1972). Tipologías de viviendas racionalizadas $1966-1972$

2
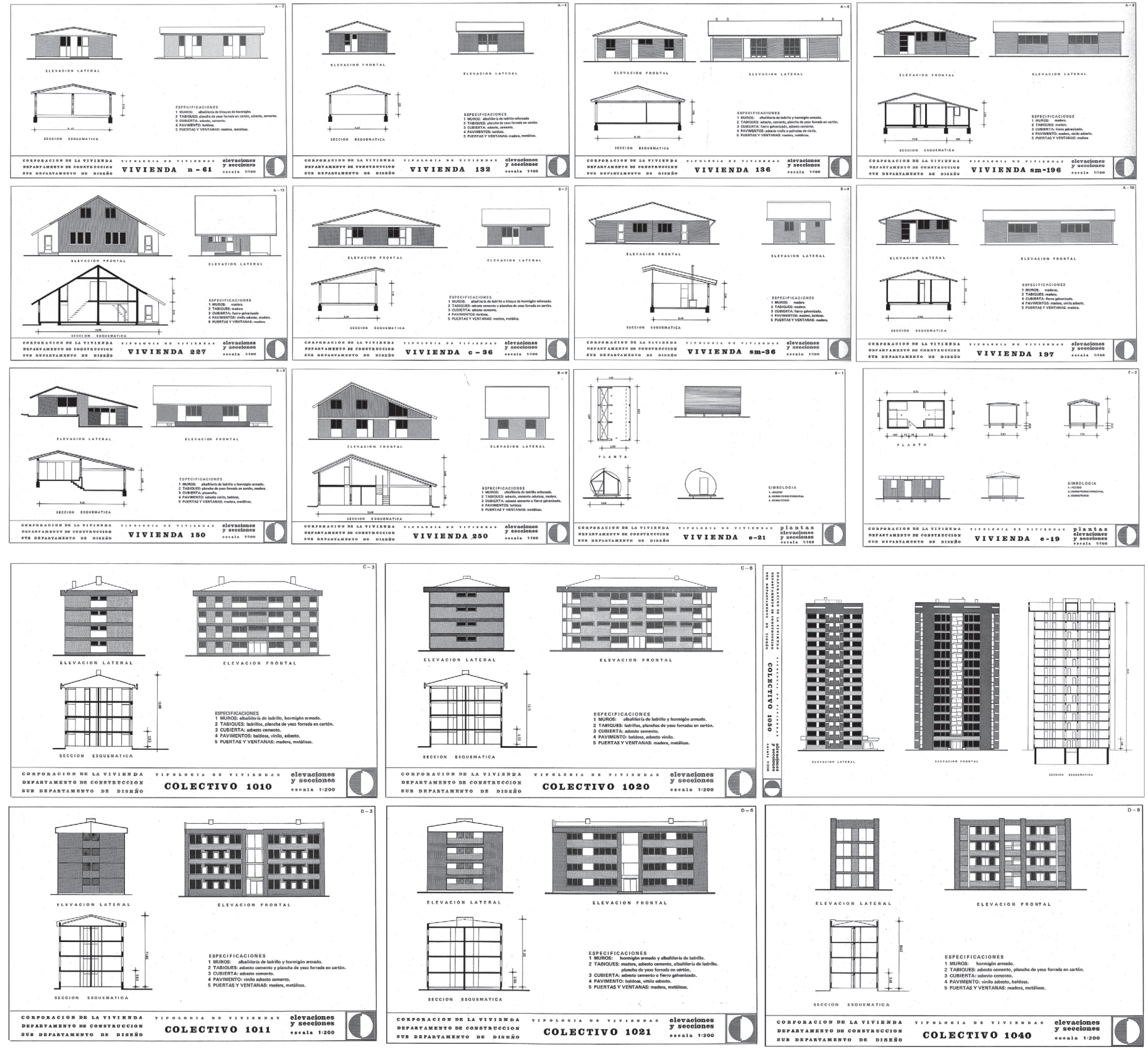
esquema o de una forma-base, una deducción de la formas precedentes, un lenguaje de patrones al cual recurrir. Un referente vago, suficientemente flexible para enlazar prácticas comunes a través de múltiples versiones locales en el tiempo, que es lo que recoge Deplazes al explicar los ejercicios de caja como iteraciones de las posibilidades de una estructura básica y común a todas las obras realizadas, una sombra similar que vincula como conjunto a todas ellas.

Por su parte, Walter Gropius elige hablar de standards para identificar estas formas replicadas, resultado de los medios de producción y de los modos de vivir de una época, "síntesis de las mejores aportaciones individuales para solucionar determinados problemas repetitivos" (Benévolo, 1963: 883). Es la línea que también sigue el influyente Arte de Proyectar en Arquitectura de Ernst Neufert (1995), texto con el que la Bauhaus organiza como método proyectual su modelo de estandarización de los efectos espaciales de movimientos y prácticas corporales de humanos y objetos. Para Le Corbusier, por su parte, "la arquitectura se sostiene sobre standards (...) los standard son cosa de lógica, de análisis, de estudio escrupuloso, y se establecen sobre un problema bien planteado. La experimentación fija definitivamente el standard" (1953:31).

Pero los estándares no son persistentes, si no se revisan y renuevan se tornan inciertos, y dejan de dar explicación a los problemas situados (Lampland y Star, 2009). En el mismo sentido, las tipologías no son un instrumento estático, sino una estructura preobjetual a partir de la cual el diseño se desarrolla de forma natural (Moneo, 2015). Se transforman cuando los elementos de su estructura formal cambian, dando paso a nuevas formas tipológicas. La noción tipológica está siempre abierta al cambio, es flexible interpretativamente, resguardando su modularidad y la comunalidad de la forma, lo que permite tanto distanciarse del pasado como anticipar el futuro (Moneo, 1978; de Graaf, 2017).
De Graaf (2017) ejemplifica en las diversas aplicaciones prácticas de la caja dentro del diseño arquitectónico, tanto la flexibilidad conceptual como el sentido abstracto y preobjetual de las tipologías. La comunalidad formal de la caja, en tanto figura tipológica, da cuenta de las operaciones de estandarización, modularidad y repetición implicadas en su trabajo, en la producción de una obra arquitectónica. Por ello, para Moneo el trabajo de la arquitectura comienza por la identificación de una tipología, independiente si después ésta será destruída, transformada o respetada. En el proceso de diseño, sostiene, "se manejan los elementos de una tipología, de una estructura formal, en la situación concreta y precisa que caracteriza a la obra singular" (1978: 190).

Los momentos más intensos en la historia de la arquitectura son, para Moneo (1978), aquellos donde una nueva tipología surge, en lo que colaboran centralmente elementos externos, como la emergencia de nuevas técnicas o de nuevos requerimientos sociales. Se trata de un momento particular, que marcará la producción de las obras de arquitectura en el mediano plazo y tendrá un efecto en las formas sociomateriales de entendimiento (reflexión) de la comunidad.

Un caso singular que ejemplifica los modos de ser tipológicos es lo ocurrido con los diseños CORVI cuya elaboración en 1966 fue organizada en torno a un sentido modernizador de formas y programas, y a un sentido racionalizador en el uso de los recursos constructivos, por su vicepresidente ejecutivo, el arquitecto Héctor Valdés Phillips. Esto da pie a un ejercicio de taller extendido, que convoca al conjunto de los equipos de diseño, que sincretiza la obra anterior en principios que se denominan racionalizados, y los proyectan en treinta y ocho tipologías de casas, bloques y edificios en altura que son recogidos posteriormente en el documento "Tipologías de viviendas racionalizadas 19661972" (CORVI, 1972) (FIgURA 2). No todos los diseños elaborados como tipologías e incluidos en el texto fueron finalmente construidos, pero claramente su sentido era orientar procesos de diseño y construcción futuros, lo que sirvió como referencia para la estandarización de las unidades de vivienda posteriores, a nivel de forma y de programa.

\section{PRACTICIDAD: UN MODO DE COOPERACIÓN}

Consideradas relacionalmente, las tipologías arquitectónicas constituyen un tipo de acuerdo objetual que permite que diferentes colectivos trabajen juntos sin la necesidad de llegar a consensos entre ellos, como ocurre en los procesos de diseño, de edificación y de habitación de lo construido. Tal como otros objetos con similares funciones, las tipologías surgen en respuesta a necesidades y requisitos de información y trabajo percibidos localmente por los actores y grupos involucrados en situaciones de cooperación. Son objetos modulares, flexibles interpretativa y materialmente desplazables, lo que les ayuda a organizar relaciones infraestructurales entre actores y comunidades de prácticas diferentes, y dar forma a un esquema político particular donde la especulación sobre otros actores requiere de información que, aunque imprecisa, debe ser lo suficientemente buena como para anticipar las prácticas, relaciones y asociaciones posibles (Lampland y Star, 2009).

La noción de objeto en este contexto semántico tiene un sentido informático, pragmático y material. Susan Leigh Star (2010) propone que objetos como las tipologías son algo que los actores reconocen como espacio e instrumento, de manera que su propia forma deriva de la acción en que son involucrados, son materia de la acción. Estos objetos, que funcionan como un espacio informático y relacional compartido entre más de una comunidad de prácticas y que operan en función del intercambio entre éstas, terminan siendo parte de sus repertorios comunes. Esto ocurre con los repositorios, tipos ideales, límites coincidentes y con formas estandarizadas que Susan Leigh Star y James Greisemer (1989) denominan objetos frontera 
debido la posición relacional antes descrita. No requieren para ello ser precisos en la descripción de detalles de ninguna cosa o localidad, lo que caracteriza a este tipo de objetos es la función infraestructural, en cuanto posibilitan diversas prácticas de cooperación.

Las tipologías arquitectónicas, al igual que los tipos ideales, son abstracciones o representaciones suficientemente vagas que no describen ninguna obra en particular, pero son útiles para diferenciar entre conjuntos de ellas. Actúan como objetos frontera, flexibles interpretativamente a más de una comunidad de prácticas, modularmente compuestos y permeables a efectos de escala, lo que permite que sean útiles para la cooperación en diversas situaciones. Son efectivas para poner de acuerdo con actores de comunidades expertas y no expertas en las situaciones de enseñanza, de venta inmobiliaria o propaganda gubernamental, a actores de comunidades diferentes en las situaciones de edificación y de zonificación, y/o para explicar problemas de gobernanza sociotécnica asociadas a la forma arquitectónica, como ocurre en las situaciones de gobierno de escala urbana. En cada uno de esos escenarios, las tipologías actúan de forma adaptable, mostrándose suficientemente buenas para lidiar con las contingencias locales de la cooperación, de manera que también pueden ser vistas como un conjunto de arreglos de trabajo entre los actores y comunidades de prácticas diferentes que necesitan de medios para entenderse y trabajar juntos.

De hecho, es posible que cada comunidad de prácticas y que cada grupo local las trabajen de manera diferente, a lo cual colabora su identidad vaga. Casas, bloques y edificios en altura pueden ser objetivo de diferentes operaciones de estandarización y no por ello logran ser estandarizadas complemente. Todos los actores entienden de qué se trata el término casa y a la vez todos las imaginan y las reproducen de manera diferente: las tipologías tienen formas variantes. Los grupos que cooperan sin consenso abordan el proceso de ida y vuelta entre las diferentes variaciones tipológicas porque lo que prima es el sustrato estructural al que apela esa imaginería.

Por su parte, la noción de frontera caracteriza bien la intencionalidad que estructura este lenguaje de cooperación. Enfatiza la presencia de coordinaciones en escenarios sin consenso, alojadas en formatos materiales. Aclara que su heterogeneidad epistemológica y su flexibilidad interpretativa no son simples cualidades, sino condiciones prácticas de tales funciones, por tanto, así como cualquier grupo de obras no constituye una tipología arquitectónica, no todos los objetos capaces de intermediar la cooperación corresponden a objetos frontera. Tampoco es el equipamiento epistemológico lo que las constituye en cuanto tal, como propone Vinck (2009), puesto que ello cerraría la posibilidad del objeto de ser flexible interpretativamente, enlazándolo a una comunidad de prácticas determinada más que dotándolo de la posibilidad de articularse como espacio común entre comunidades diferentes. Por ello la discusión sobre las tipologías persiste como proceso y enlaza arreglos de practicantes situados en lugares y tiempos diferentes.

Lo anterior reafirma que los objetos arquitectónicos son parte de un modo de pensar y hacer sobre el mundo, una manera de tejer socialmente la realidad a partir de un mundo de la vida determinado. Pero en esto no están solos, requieren de aliados políticos, epistemológicos y objetuales para ser realizados. Necesitan de instrumentos que les ayuden en el trabajo de transformar la realidad, lo que les constituye también en un modo de operar colectivo sobre el mundo existente y sobre el mundo posible. Una disciplina de las acciones, que les permiten actuar sobre los actores y sus diferencias.

Las tipologías son parte de estos repertorios. Apuntan, a prácticas preobjetuales cuyo trabajo político es cómo definir los parámetros de diferencia y similitud entre diferentes objetos y cómo se colabora en su concreción. De manera diferente a las políticas del quién, que tratan sobre las personas y sus derechos, individuales y colectivos; y a las políticas del qué, que versan sobre los modos en que las prácticas generan realidades; las políticas del cómo, atienden a las formas en que las realidades se enactan en prácticas, a cómo esas prácticas reconocen y manejan las diferencias entre actores y comunidades, y a cómo las diferencias pueden ser mejor manejadas (Law y Joks, 2018).

\section{CONCLUSIÓN. ORDEN Y PRACTICIDAD}

Los procesos de orden y categorización de las obras de una comunidad de prácticas, como ocurre con la arquitectura, han sido observados utilizando diversos conceptos objetuales. Dentro de éstos cabe considerar a los mutables inmóviles (Latour, 1987; Guggenheim, 2016), los puntos de paso obligado (Callon, 1984), los objetos epistémicos (Knorr Cetina, 1997), y los objetos intermediadores, que han sido formulaciones útiles para describir los modos como objetos materiales colaboran en la organización de lo social en ecologías sociotécnicas (Vinck, 2009).

Vistas desde el modelo de objetos frontera (Star, 2010), las tipologías arquitectónicas constituyen un tipo de arreglo sociomaterial que permite que diferentes grupos trabajen juntos sin consenso. Surgen en respuesta a necesidades y requisitos de información y trabajo percibidos localmente por los actores y grupos involucrados en situaciones de cooperación, como son el diseño, construcción, venta y habitación de edificios, así como también en la planificación urbana. Lo que aporta la noción de frontera a la descripción de la acción de las tipologías en la comunidad de prácticas de la arquitectura tiene que ver con situar objetualmente las relaciones de cooperación, aún sean agenciadas en una abstracción, y con situar espacialmente la identidad de la comunidad de práctica, aún cuando el espacio en cuestión no corresponda a un interior, un borde o un exterior. En este sentido, el espacio de cooperación demuestra la comunidad sin ser necesariamente un linde o un límite con otra, sino una configuración cooperativa donde es posible trabajar juntos. 
La metáfora de la frontera es útil para entender cómo funciona la cohesión en los entornos socio técnicos implicados en las prácticas del habitar urbano. Los estándares y las tipologías constituyen fronteras que colaboran en mantener unidas las prácticas de una comunidad, son un instrumento de cohesión, que permite sostener una reflexión sociomaterial en el tiempo. El aporte de la noción de frontera radica en su capacidad para producir una inversión infraestructural, esto es, poner frente a la observación el entramado de elementos sociomateriales que, como el diseño, comúnmente permanecen detrás de la acción y que la habilitan como soportes de esta. Star y Lampland (2009), señalan que este trabajo es a menudo invisible en la imagen general de los procesos o eliminado de la descripción de estos. También lo son las mismas tipologías en cuanto espacios de trabajo de las comunidades, donde conocimiento experto y experiencia se intercambian entre actores de diferentes épocas, funciones y comunidades de prácticas que buscan cooperar entre sí; en tanto espacios donde se negocian modos de dimensionar y ordenar el mundo y sus interacciones. En este sentido, las tipologías arquitectónicas no solo son interesantes como arreglos espaciales comunes entre comunidades de prácticas diferentes, sino también como procedimientos de orden y verdad, como órdenes relacionales desplegados en la forma de un diseño.

Para describir la utilidad heurística del estudio de estos objetos, Star y Lampland (2009) aluden a hilos que cabe desentrañar para hacer visible y animado el momento en que una estructura se convierte en una estructura y en el que un actor o un agente se hacen parte de esa historia. El orden tipológico es tanto un modo de pensar las prácticas a través de sus similitudes estructurales como un modo de aprender, comunicar y desplazar soluciones formales persistentes entre comunidades de prácticas deslocalizadas. El ejercicio detrás del objeto tipológico no cierra nunca del todo. Permite especular y hacer cambios se posibilita a sí mismo, permite pensar el significado de la diferencia. Pensar el significado de la predictibilidad del mundo a partir de la iteración de la práctica y el ejercicio tipológico, no solo es un modo de ordenar un mundo desordenado, también es una estrategia para que el orden mismo sea una posibilidad real, táctilmente hablando. Una práctica tan sincrética como especulativa, en la medida que articula los aspectos comunes de un conjunto de obras en un canon, que orienta otras prácticas que lo exploran e innovan, y que producen otras obras diferentes.

La practicidad de los tres modos descritos radica en que las tipologías resultan figuras "suficientemente buenas" para que los actores que participan de la comunidad de prácticas de la arquitectura puedan especular sobre formas de orden, proyectos y alianzas con otros actores, tanto dentro como fuera de ésta. De esta manera, las tipologías no sólo son útiles para "hacer política" en torno a las obras de arquitectura y para tener un efecto relacional en otros actores, también constituyen un "espacio político" donde actores y comunidades de prácticas distintas pueden cooperar entre sí, $\sin$ tener que negociar sus diferencias. Esto último puede ser particularmente interesante si se considera que políticas públicas acerca del habitar pueden inspirarse desde nociones tipológicas $y$, por tanto, funcionalmente relacionales, y no solo económicas, poniendo una política de cohesión como motor de las prácticas distributivas.

\section{REFERENCIAS BIBLIOGRÁFICAS}

Argan, G.C. (1983). Tipología. Summarios 71: 2-14. Bauman, Z. (2003). Comunidad. En busca de seguridad en un mundo hostil. Buenos Aires: Siglo XXI Editores.

Benévolo, L. (1963). Historia de la Arquitectura Moderna. Madrid: Taurus

Callon, M. (1984). "Some Elements of A Sociology of Translation: Domestication of the Scallops and the Fishermen of St Brieuc Bay". The Sociological Review. 32. DOI:10.1111/j.1467-954X.1984 tb00113.x.
Collado, A. (2017). Modernidad y tipología. ARQUISUR Revista, 7(11), 64-79. https://doi. org/10.14409/ar.v0i1 1.6677

CORVI (1972). Tipologías de viviendas racionalizadas 1966-1972. Valparaíso: Subdepartamento de Diseño CORVI.

De Graaf, R. (2017). Four Walls and a Roof: The Complex Nature of a Simple Profession. Cambridge: Harvard University Press

Delfante, Ch. (2006). Gran Historia de la Ciudad. De Mesopotamia a Estados Unidos. Madrid: Adaba Editores

Durand, J.N.L. (2000). Précis of the Lectures on Architecture. Los Angeles: The Getty Research Institute

Guggenheim, M. (2014). From Prototyping to Allotyping. The invention of change of use and the crisis of building types. Journal of Cultural Economy, 7(4): 411 -433. http://dx.doi.org/10.10 80/17530350.2013.858060

Guggenheim, M. (2016). 'Im/Mutable Im/Mobiles. From the Socio-Materiality of Cities towards a Differential Cosmopolitics'. En, Andres Blok y Ignacio Farías, (Eds). Urban Cosmopolitics. Agencements, Assemblies, Atmosphere. https:// doi.org/10.4324/9781315748177

Hughes, F (2014). The architecture of error. Matter, measure, and the misadventures of precision. Cambridge: The MIT Press

Hughes, F (2016). The architect. Reconstructing her practice. Cambridge: The MIT Press

Ingold, T. (2013). Making: Anthropology, Archaeology, Art and Architecture. London: Routledge

Ingold, T. (2017). What if the city were an ocean and its buildings ships? In S. V. Bradley (Ed.), The Evergreen: A New Season in the North (Vol. 3, pp. 11-18). Edinburgh: The Word Bank.

Knorr Cetina, K. (1997). Sociality with Objects. Theory, Culture \& Society, 14(4), 1-30. doi:10.1177/026327697014004001

Lampland, M. y Star, S. L. (Ed) (2008). Standards and Their Stories: How Quantifying, Classifying, and Formalizing Practices Shape Everyday Life. Cornell University Press

Latour, B. (1987). Science in Action: How to Follow Scientists and Engineers Through Society. Cambridge: Harvard University Press. 
Law, J., \& Joks, S. (2018). Indigeneity, Science, and Difference. Science, Technology, \& Human Values, 016224391879394. doi: $10.1177 / 0162243918793942$

Le Corbusier (1953). El Modulor. Ensayo sobre una medida armónica a la escala humana aplicable universalmente a la arquitectura y a la mecánica. Buenos Aires: Editorial Poseidon

Moneo, R. (1978). On typology. En, Oppositions 13: 22-45. Cambridge: The MIT Press.

Moneo, R. (2015) Typology in the context of three projects: San Sebastian, Lacua, Aranjuez, The Journal of Architecture, 20:6, 1067-1087, DOI: 10.1080/13602365.2015.1116347

Neufert, E. (1995). Arte de proyectar en Arquitectura. Fundamentos, normas y prescripciones sobre construcción, dimensiones de edificios, locales y utensilios, instalaciones, distribución y programas de necesidades. Barcelona: Gustavo Gili
Quintanilla, J. (2018). Cuidar el tiempo, hacerse el tiempo, espaciar el tiempo. ARQ (Santiago), (100), 94-105. https://dx.doi.org/10.4067/ S0717-69962018000300094

Rossi, A. (2015). La Arquitectura de la Ciudad. Barcelona: Gustavo Gili.

Star, S. L. (2010). This is Not a Boundary Object: Reflections on the Origin of a Concept. Science, Technology, \& Human Values, 35(5), 601-617. doi:10.1177/0162243910377624

Star, S. L. y Griesemer, J. (1989). Institutional Ecology, 'Translations' and Boundary Objects: Amateurs and Professionals in Berkeley's Museum of Vertebrate Zoology, 1907-39. Social Studies of Science, 19(3), 387-420. www.jstor.org/ stable/285080

Sullivan, L. H. (1896) "The tall office building artistically considered". En Lippincott's Magazine 57, March, 1896. Philadelphia Pp. 403-409
Thévenot, L. (2019). How Does Politics Take Closeness into Account? Returns from Russia. International Journal of Politics, Culture, and Society. doi:10.1007/s10767-019-9322-5

Tönnies, F. (1947). Comunidad y Sociedad. Buenos Aires: Editorial Losada

Vinck, D. (2009). De l'objet intermédiaire à l'objetfrontière: Vers la prise en compte du travail d'équipement. Revue d'anthropologie des connaissances, vol. 3, 1 (1), 51-72. doi:10.3917/ rac.006.0051.

Zayas, M. B. (2015). Evolución de la tipología arquitectónica y caracterización paisajística de los grandes equipamientos urbanos. BAETICA. Estudios De Historia Moderna Y Contemporánea, (34), 103-125. https://doi.org/10.24310/ BAETICA.2012.v0i34.78 